\title{
Análisis
}

\section{Software libre en repositorios de e-información}

\author{
Por Ricard de-la-Vega-Sivera
}

\begin{abstract}
Resumen: Las bibliotecas universitarias no sólo gestionan aquellos recursos electrónicos generados por editoriales comerciales, sino que también, y cada vez más, documentos fruto de actividades docentes e investigadoras de sus propias instituciones. Es función de las bibliotecas difundir estos materiales en forma electrónica, pasando previamente por las fases de almacenamiento y organización, así como garantizar su preservación. La forma de hacerlo es mediante los repositorios de e-información. En este artículo se pretende dar una rápida visión de los diferentes programas de software libre para el desarrollo de repositorios, centrándose en aquellos que se han usado para la elaboración de depósitos cooperativos catalanes. También se repasan algunas características deseables de estos sistemas, como son el uso de estándares abiertos de comunicación entre repositorios, las estrategias de preservación de los contenidos digitales y las comunidades de usuarios.
\end{abstract}

Palabras clave: Repositorios, E-ciencia, Acceso abierto, Software libre, Investigación.

\section{Title: Free software in e-information repositories}

Abstract: University libraries manage not only commercial electronic resources, but also documents generated by research and educational activities at their own institutions. Libraries are committed to disseminating these electronic documents, which usually requires that they compile and store them as well as guaranteeing their preservation. The best way to achieve this objective is e-information repositories. We briefly present an overview of the free software available to develop repositories, with a special focus on the software used to build Catalan cooperative repositories. We also review desireable system characteristics, such as the use of open communication standards between repositories, preservation strategies for digital content, and user communities.

Keywords: Repositories, E-science, Open access, Free software, Research.

De-la-Vega-Sivera, Ricard . "Software libre en repositorios de e-información". En: El profesional de la información, 2008, enero-febrero, v. 17, n. 1, pp. 49-55.

DOI: 10.3145/epi.2008.ene.05

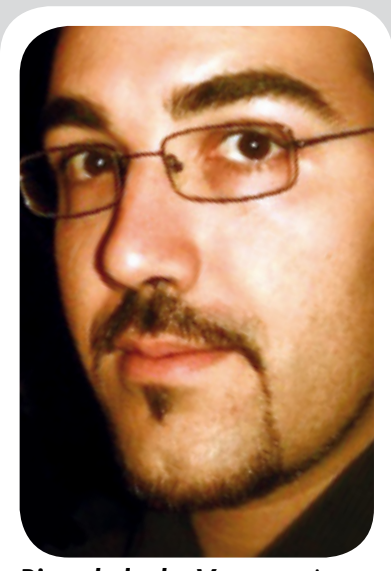

Ricard de la Vega es ingeniero técnico en informática de gestión por la Universidad Politécnica de Catalunya y ha realizado un postgrado de tecnologías de la comunicación. Ha sido formador de títulos oficiales de empresas del sector informático y desarrollador de proyectos web antes de entrar a formar parte del Departamento de Sistemas del Centro de Supercomputación de Catalunya, donde ahora coordina el área de repositorios y portales universitarios.

\section{Introducción}

LAS BIBLIOTECAS SON ORGANIZACIONES QUE ORDENAN y almacenan colecciones de documentos, tanto impresos como electrónicos, para su preservación y consulta. Concretamente, las bibliotecas universitarias no sólo gestionan aquellas fuentes de información electrónicas generadas por editoriales comerciales, sino que también, y cada vez más, documentos fruto de actividades docentes e investigadoras de sus propias instituciones (Anglada et al, 2005). Todo aquello que hace unos años se generaba sobre pa- pel, hoy en día se realiza de manera digital. Es función de las bibliotecas difundir estos documentos en forma electrónica, pasando previamente por las fases de almacenamiento y organización, así como garantizar su preservación. El procedimiento para hacerlo es mediante los repositorios de e-información.

Estos repositorios son una parte importante del movimiento del acceso abierto (Open access), que desde hace unos años apuesta por una nueva manera de difusión del conocimiento. Las contribuciones del acceso abierto han de cumplir, según la Declaración de Berlín, dos condiciones. Por un lado, los autores y los que retienen el derecho sobre las colaboraciones han de garantizar a todos los usuarios el derecho al acceso abierto con licencia para copiar, usar, difundir, transmitir y exponer los trabajos públicamente con cualquier propósito responsable, a cambio de mencionar como es debido la autoría. Por otro lado, una versión completa de esta obra se tendrá que depositar en formato electrónico en un sitio en línea, esto es, en un repositorio.

En este sentido, el Centre de Supercomputació de Catalunya 


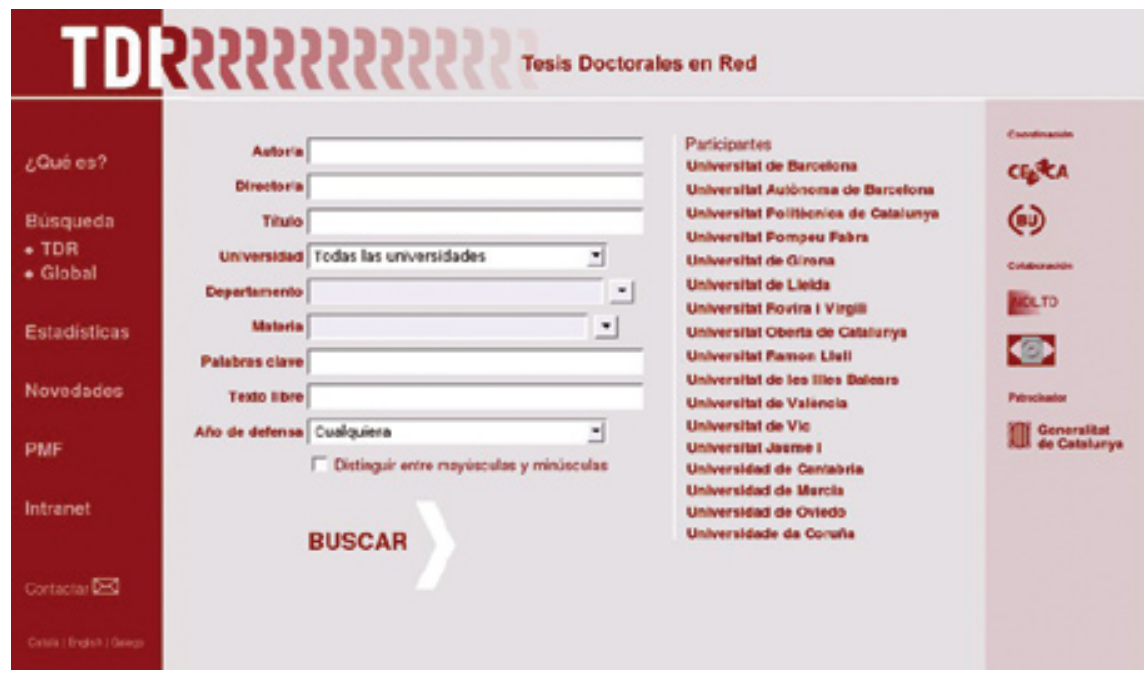

Tesis Doctorales en Red (TDR)

(Cesca) y el Consorci de Biblioteques Universitàries de Catalunya $(C B U C)$ empezaron en 1999 un repositorio cooperativo para almacenar en formato digital el texto completo de tesis doctorales leídas en universidades de Cataluña y de otras comunidades autónomas para difundirlas mundialmente, en acceso abierto, preservando los derechos de propiedad intelectual de sus autores. Este repositorio, llamado Tesis Doctorales en Red (TDR) comenzó a funcionar hace 7 años y hoy en día está plenamente consolidado.

http://www.tesisenred.net

Los resultados positivos de esta iniciativa hicieron posible que la Generalitat de Catalunya patrocinase dos nuevos repositorios en acceso abierto: Dipòsit de la Recerca de Catalunya (Recercat) y Revistes Catalanes amb Accés Obert $(R A C O)$. El primero es un depósito cooperativo de literatura de investigación de las universidades y centros de investigación de Cataluña: artículos aún no publicados, actas, informes de investigación, etc. El segundo permite consultar en acceso abierto los artículos completos de revistas científicas, culturales y eruditas catalanas.

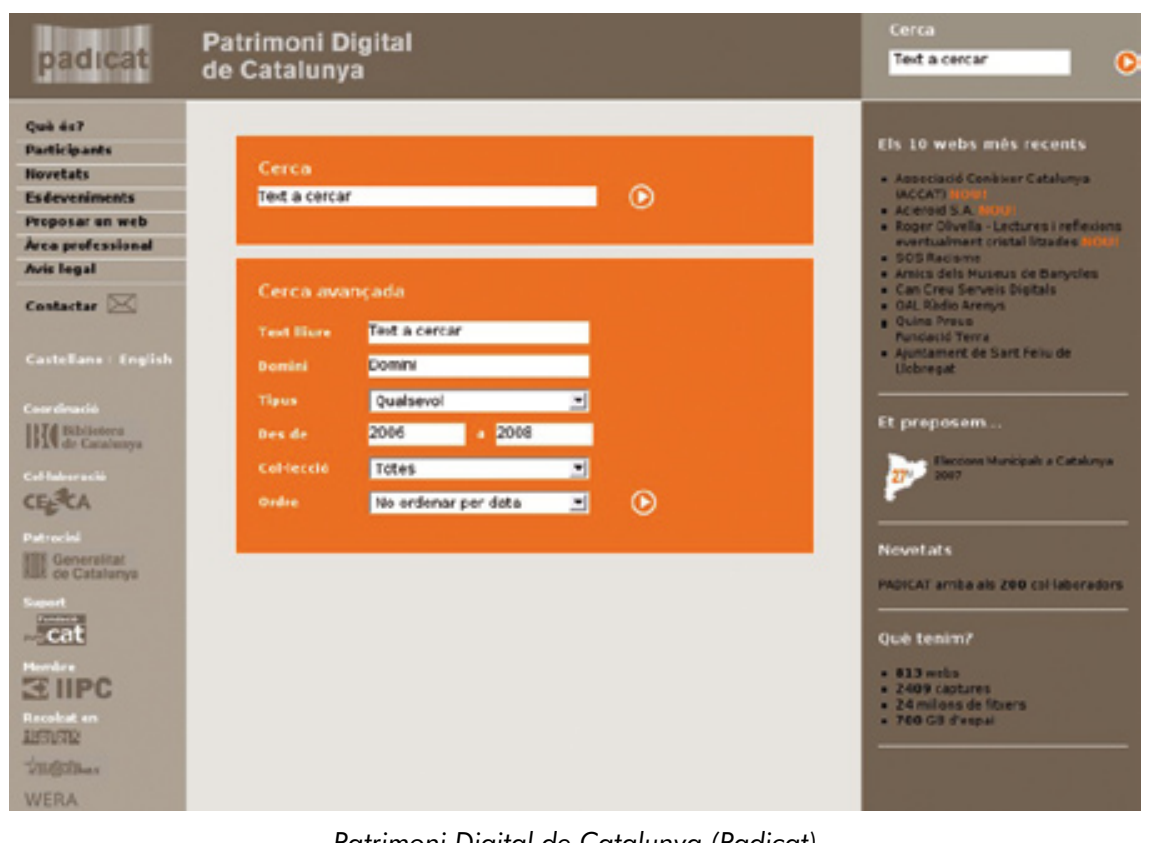

\section{http://www.recercat.net}

http://www.raco.cat

En 2006 la Biblioteca de Catalunya inició otro ambicioso repositorio en colaboración con el Cesca: Patrimoni Digital de Catalunya (Padicat)(Llueca, 2006). Éste se estableció para garantizar el acceso permanente a la producción de webs catalanas. De esta manera, se implementa el tradicional ciclo de los documentos (compilación, gestión, preservación y difusión) para el material bibliográfico publicado en internet.

\section{http://www.padi.cat}

Los cuatro repositorios tienen algunas características comunes: son de acceso abierto; están desarrollados con software libre; cumplen con el protocolo de interoperatibilidad de la Open Archives Initiative (OAI) para compartir metadatos; y están construidos colectivamente, pudiendo adoptar procedimientos comunes, compartir costes de desarrollo y gestión, así como permitir una mayor visibilidad de los contenidos.

En este trabajo se pretende dar una rápida visión de algunos programas de software libre existentes para el desarrollo de repositorios, centrándose en los que se han usado para la elaboración de los mencionados depósitos cooperativos (Huguet et al, 2007). También se repasan algunas características deseables de estos sistemas, como son el uso de estándares abiertos de comunicación entre ellos, estrategias de preservación de los contenidos digitales o las comunidades de usuarios.

\section{Software libre para repositorios}

La Universidad de Nottingham mantiene un listado (dotado con un potente buscador) de repositorios de contenidos en acceso abierto, dentro del proyecto OpenDOAR. Aquí puede observarse que la tendencia es desarrollarlos con aplicaciones 
"La principal ventaja que el software libre ha aportado a los repositorios es la adaptabilidad"

libres. De los 689 depósitos que están en estos momentos inventariados y especifican el software con el que están realizados, más del $80 \%$ son de software libre (30\% DSpace, $28 \%$ EPrints, $7 \%$ OPUS, $2 \%$ ETD$d b$, etc.).

\section{http://www.opendoar.org}

Las ventajas que este tipo de software ha aportado a los repositorios son muchas, pero sin lugar a dudas, la principal ha sido la adaptabilidad. Puede que haya principios básicos genéricos, pero cada proyecto es singular. En los cuatro repositorios cooperativos citados se han realizado mejoras que han acercado el software a los requisitos que se han marcado para cada uno de ellos. Aparte de la adaptabilidad y de la gratuidad, los depósitos también se han beneficiado de otras ventajas (Mas, 2006), como el escrutinio público, donde el código está sometido a un proceso de revisión que aporta dinamismo a la corrección de errores, o la independencia del proveedor, hecho que garantiza su continuidad teórica.

A principios de 2004 se formó un grupo de trabajo para acordar la selección conjunta de un software libre para gestionar repositorios electrónicos de objetos digitales (Borràs et al, 2006). El estudio se centró en los programas ARNO, Cdsware, DSpace, EPrints, Fedora, $i$-TOR y MyCore, todos ellos propuestos en un informe del Open Society Institute (2004). Se trataba de un grupo mixto entre bibliotecarios e informáticos del $C B U C$ y algunas universidades catalanas. Se quiso una selección conjunta para crear repositorios institucionales con el mismo programa, y de esta manera poder crear un grupo de usuarios

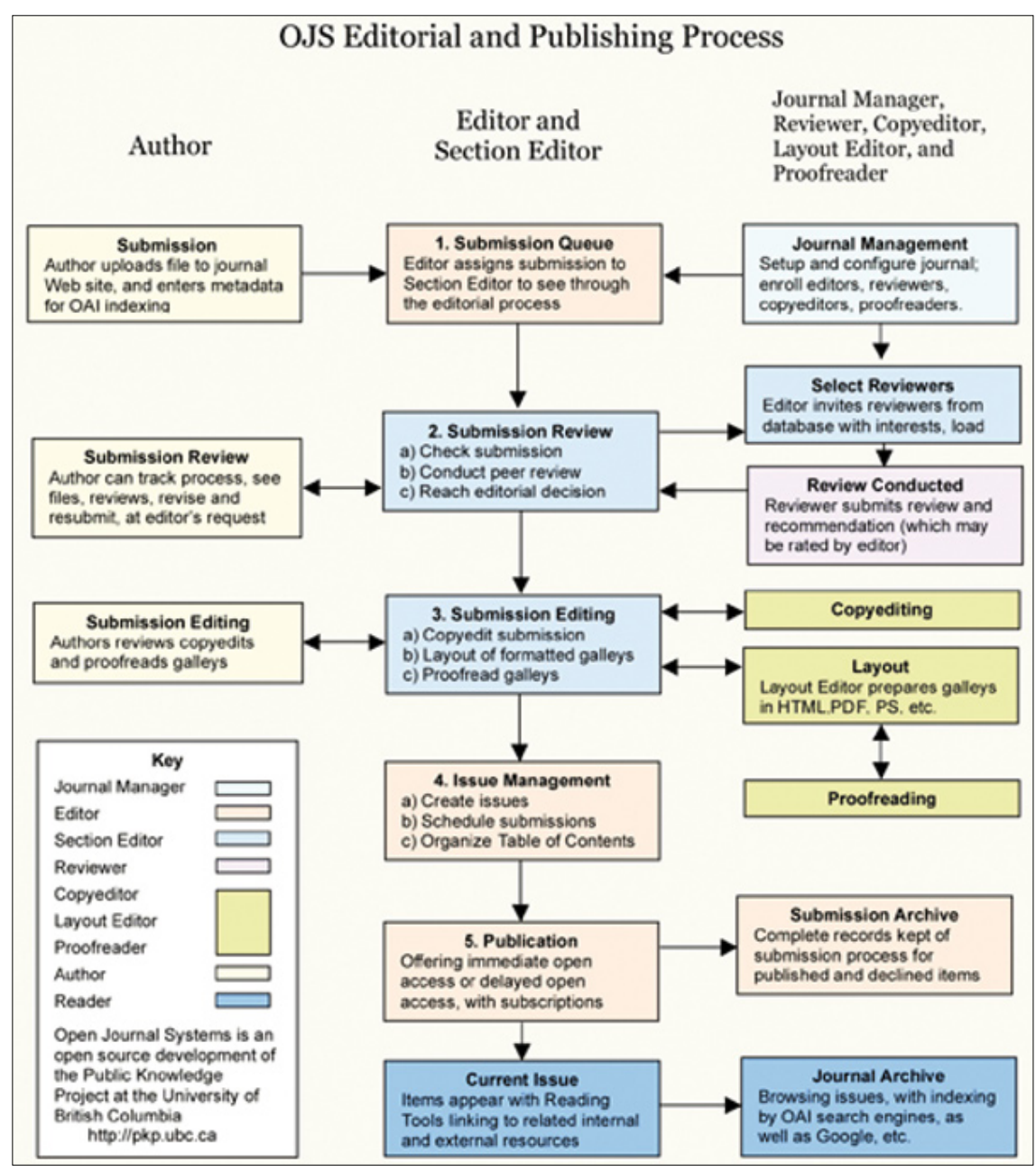

Open Journal Systems (OJS)

que colaborase en la solución de problemáticas comunes. Finalmente se escogió DSpace, que describiremos más adelante, por su extenso uso a nivel internacional, por ser un sistema acabado que podía usarse inmediatamente, por su alto nivel de prestaciones y por su facilidad de uso, tanto para el usuario final como para su administrador.

En la literatura pueden hallarse otros trabajos comparativos sobre aplicaciones para el desarrollo de repositorios. Jesús Tramullas y Piedad Garrido hacen un extenso inventario (Tramullas-Garrido, 2006: 171-181) donde después proponen un modelo para evaluar las prestaciones. Para el caso concreto de revistas electrónicas existen programas que además de poder depositar los artículos, sirven también para realizar todo el proceso de revisión por pares, edición y publicación. En el proceso de selección de $R A C O$ fueron estudiados DSpace+CWIS, uPortal, CON$T E N d m$ y Lodel y finalmente se escogió Open Journal Systems (OJS), al tratarse de la opción más completa y acabada para revistas.

De todos los ejemplos mencionados, se describen más detalladamente los que se han usado en la elaboración de repositorios cooperativos catalanes. Para TDR se adaptó el programa Electronic Theses and Dissertations (ETD$d b$ ) de la Universidad de Virginia Tech; para Recercat, se seleccionó DSpace; para RACO se usa OJS; y para Padicat conviven el recolector Heritrix, el indexador y buscador NutchWAX y la interfaz de consulta WERA y Wayback.

De hecho, TDR inició su camino como base de datos, pues en 1999 
todavía ni la Scholarly Publishing and Academic Resources Coalition (Sparc) había definido los repositorios institucionales. El programa escogido para su implementación, $E T D-d b$, fue una buena elección en su momento pues cumplía con los requisitos del repositorio y era el único. Hoy en día todavía cumple satisfactoriamente con su misión; sin embargo, la evolución del software en comparación con otros, por ejemplo DSpace, ha sido notablemente más lenta, hecho por el cual se está estudiando su sustitución a medio plazo. A continuación nos centramos por lo tanto en DSpace como aplicación para repositorios de propósito general; en OJS para la edición y publicación de revistas; y en los diferentes programas agrupados bajo el paraguas de Heritrix para repositorios de sitios web.

\section{http://etd.vt.edu}

\section{DSpace}

Es un programa de código abierto creado por el Massachusetts Institute of Technology (MIT) y Hewlett Packard (HP). Se trata de un repositorio digital innovador que captura, almacena, indiza, preserva y redistribuye los resultados de la investigación de una organización. Existen 275 instalaciones en 45 países y permite búsquedas por autor, título, universidad, centro de investigación, etc. También es posible suscribirse al servicio de alertas de una colección y otros elementos propios de la biblioteca 2.0.

El hecho de ser de código abierto ha permitido adaptarlo a los requisitos de Recercat: personalizar la presentación; normalizar la búsqueda con diacríticos; adaptar la clasificación por materias de los documentos según la CDU; establecer un control de autoridades para los nombres de los autores y las instituciones; implementar las búsquedas por tipo de documento, y realizar un módulo de estadísticas personalizado, pues los diferentes pluggins que se ofrecen con DSpa-

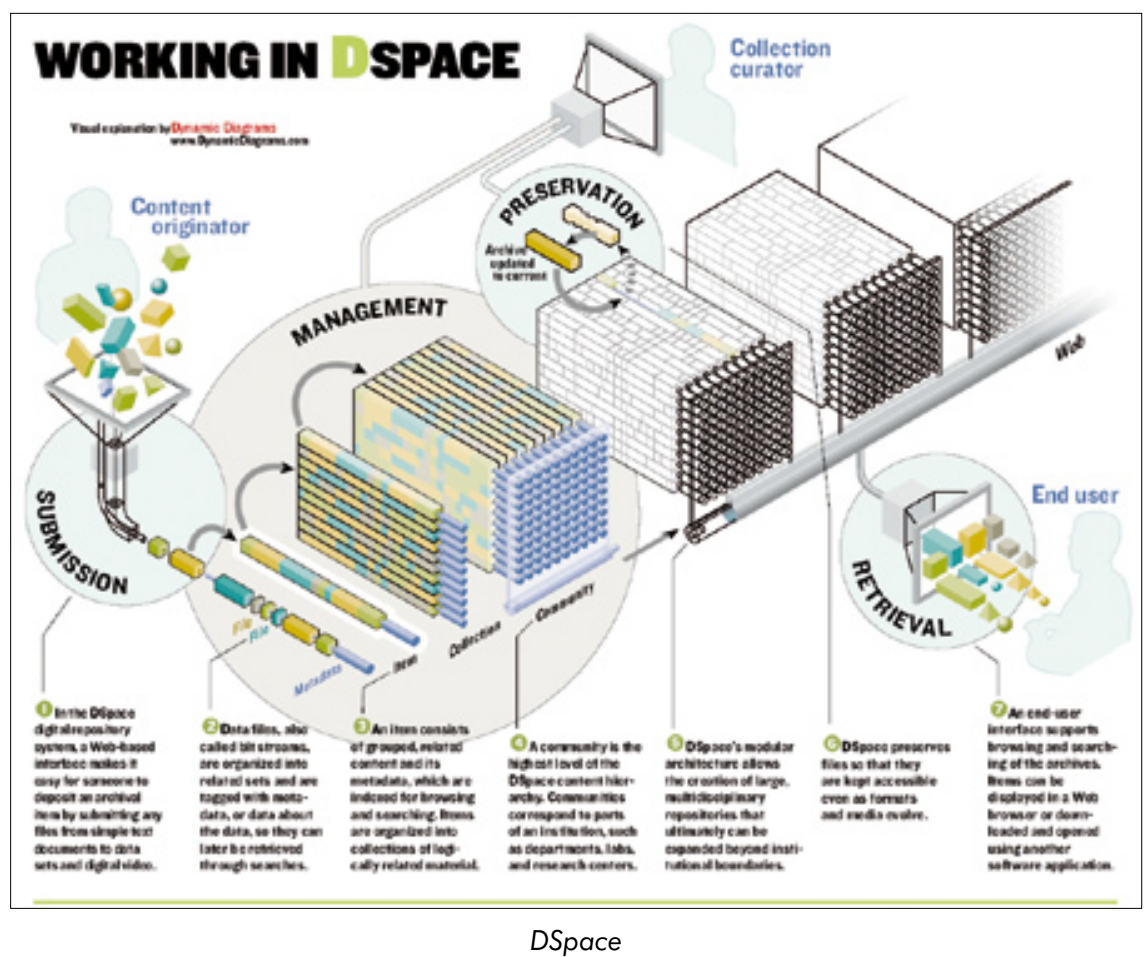

ce no cumplían con los objetivos deseados para el proyecto. También se resolvieron errores en el módulo de importación de metadatos en los casos en los que se producían modificaciones. Y con todo esto se ha contribuido a la comunidad DSpa$c e$, tomando un papel activo en el movimiento del software libre.

\section{http://www.dspace.org}

\section{OJS}

Es un programa de código abierto desarrollado por el Public Knowledge Project (PKP) con el objetivo de promover el acceso a la investigación, facilitando la administración y publicación de revistas científicas. Permite realizar todo el proceso de revisión por pares y publicación de revistas. En su vertiente de repositorio permite búsquedas por autor, título de artículo, título de publicación o tema. Para cada artículo es posible consultar la bibliografía, las estadísticas, el metadato que lo describe, recomendar su lectura a colegas, etc.

Para $R A C O$, aparte de normalizar las búsquedas con diacríticos y traducirlo al catalán, se han llevado a cabo dos nuevos módulos. El primero, para implementar la clasifica- ción temática de las revistas (según la CDU), puesto que la aplicación estaba más orientada a artículos que a revistas, y características como éstas no se encontraban disponibles. El segundo módulo simplifica el proceso de publicación para aquellas revistas que no tengan la suficiente estructura o volumen como para atacar el detallado proceso de publicación de OJS.

\section{http://pkp.sfu.ca/ojs}

\section{Heritrix, NutchWAX, Wera,}

\section{Wayback, etc.} ro en España, pero las aplicaciones usadas por proyectos similares internacionales son bastante comunes. Un conjunto de programas informáticos permite recoger, almacenar, organizar, preservar y ofrecer acceso permanente a las webs publicadas en la Red. Heritrix es el recolector de Internet Archive y su misión es arrastrar y almacenar en archivos comprimidos las páginas de las webs seleccionadas. NutchWAX (basado en Google) genera los índices que más tarde serán usados para las búsquedas. Y finalmente, Web Archive Access (WERA) es una solución libre para buscar y navegar por las
Padicat es un proyecto pione- 
colecciones de documentos de los distintos sitios web archivados.

Se ha evaluado, y pronto estará también en producción el buscador Wayback, que a diferencia de WERA sólo permite las búsquedas por url en lugar de a texto completo. Ambos se complementan, pues Wayback será usado para hacer búsquedas a partir de un listado catalogado de contenidos. En estos programas se están realizando también adaptaciones: para Heritrix se ha implementado un módulo de estadísticas y se está produciendo una herramienta de catalogación.

\section{http://crawler.archive.org}

http://archive-access.sourceforge.net/projects/nutch

http://archive-access.sourceforge.net/projects/wera

http://archive-access. sourceforge.net/projects/wayback

\section{Características deseables de los repositorios}

\section{Interoperabilidad}

La manera más efectiva de aumentar la visibilidad de los contenidos de los depósitos es compartiendo metadatos con otros; de esta manera los materiales se pueden dejar en local y ser buscados desde otros repositorios internacionales. Para hacer esto posible se usa un protocolo de interoperabilidad creado por la Open Archives Initiative (OAI), organismo que desarrolla y promueve estándares que se proponen facilitar la diseminación eficiente del contenido. $O A I$ tiene sus raíces en el acceso abierto y los movimientos de repositorios institucionales. Con el tiempo, sin embargo, su trabajo se ha ampliado para promover el acceso amplio a recursos digitales para la escolarización, la educación y la ciencia.

\section{http://www.openarchives.org}

Uno de estos estándares es el protocolo de $O A I$ para la recolec- ción de metadatos (OAI-PMH), un mecanismo de bajo nivel para la interoperabilidad entre repositorios. Hay dos actores posibles en el protocolo: por un lado, los proveedores de datos (data providers) son repositorios que exponen metadatos estructurados vía protocolo $O A I-P M H$. Por otro, los proveedores de servicio (service providers) efectúan peticiones OAI-PMH a los proveedores de datos para recolectar esos metadatos.

Existen ya diversas iniciativas de recolectores de metadatos. En España, desde mayo de 2006 se pueden buscar en TDR las tesis de todos los repositorios españoles que publican a través de $O A I-P M H$, convirtiéndose así en el primer recolector de tesis españolas. En la Comunidad de Madrid, e-Ciencia permite buscar entre la producción científica de sus universidades. A nivel europeo, el proyecto Driver, financiado por la Comunidad Europea, quiere recoger de la red de repositorios de los diferentes países los del ámbito de la investigación y la educación superior, e incluso ha publicado unas directrices para ello (Driver).

\section{Preservación a largo plazo de los datos}

Es un aspecto complejo, y precisamente se encargó un estudio sobre el tema para los repositorios cooperativos catalanes (Gallart; Keefer, 2007). Existen diferentes aproximaciones al problema, algunas de las cuales son resueltas por los propios programas; en otras ocasiones se necesitan aplicaciones adicionales, aunque hay que tener en cuenta además otros aspectos de carácter organizativo que nada tienen que ver con unos y ceros.

Desde el punto de vista tecnológico, algunas de las estrategias que pueden ser adoptadas son la migraciones de formatos, los controles de la persistencia de los datos (checksums) y la diversidad, ya sea en repositorios oscuros (no accesibles) o mediante dispersión geográfica, como el modelo Lots Of Copies Keep Stuff Safe (Lockss). El Open Archival Information System (OAIS), elaborado por el Consultative Committee for Space Data Systems de la NASA, ha sido adoptado por diferentes instituciones que tienen la misión explícita de preservar información, y se ha convertido en el estándar ISO 14721:2003.

La Universidad de Cambridge colabora activamente en el proyecto DSpace, y está especialmente interesada en los aspectos de preservación de recursos digitales al ser una de las bibliotecas receptoras del depósito legal de su país. En este marco está al frente del proyecto Digital Preservation Tools and Strategies, enfocado a mejorar la funcionalidad de DSpace como programa capaz de dar soporte a tareas de preservación.

\section{Comunidad de usuarios}

La robustez es garantía de continuidad del software. Al contrario de lo que sucede con los programas comerciales, en software libre no se está atado a una única empresa. Sin embargo, hay muchos programas que no llegan a buen puerto por diferencias en la comunidad, falta de recursos, cambio de inquietudes de los desarrolladores principales, etc. Afortunadamente, la mayoría de proyectos de repositorios institucionales elaborados con aplicaciones libres están siendo llevados a cabo en un entorno corporativo, principalmente gracias a fondos de investigación financiados por administraciones públicas (Chawner, 2005). Esto implica unos equipos de desarrollo estables que repercuten positivamente en la calidad de las aplicaciones resultantes. Las principales organizaciones que apoyan estos proyectos son universidades, centros de investigación y bibliotecas universitarias.

Algunas, como por ejemplo la comunidad de DSpace, han creado 
fundaciones para liderar y dar soporte al crecimiento del software. Además, se organizan eventos periódicos como el DSpace user group meeting, ocasión que sirve para poner en común todas las iniciativas que las diferentes instituciones están realizando en el programa y donde se dibuja el roadmap del producto. El último se ha realizado en octubre de 2007 en Roma.

Además de que la comunidad esté respaldada por grandes instituciones también es muy importante contar con el apoyo de grupos locales, pues muchas veces determinados problemas preocupan más a unos que a otros. Por ejemplo, DSpace es un programa de origen anglosajón y la problemática de las búsquedas correctas con diacríticos salió de un miembro del Grupo de usuarios DSpace de España $(G U D E)$ quien, como es lógico, tenía una escala diferente de urgencia por resolver el problema.

http://sod.upc.es/gude/index. php/Portada

\section{Conclusiones}

Los repositorios de e-información son una parte importante del movimiento de acceso abierto. Además, ayudan a las bibliotecas a cumplir con su misión de difundir los recursos electrónicos generados en las propias instituciones, pasando antes por las fases de almacenamiento y organización, así como de garantizar su preservación. Para poder adaptar el programa informático a los requisitos concretos del re-

\section{"Si unimos los conceptos de acceso abierto y software libre obtenemos un binomio ganador, pero si además le añadimos el concepto de estándares abiertos, obtenemos una apuesta robusta y segura"}

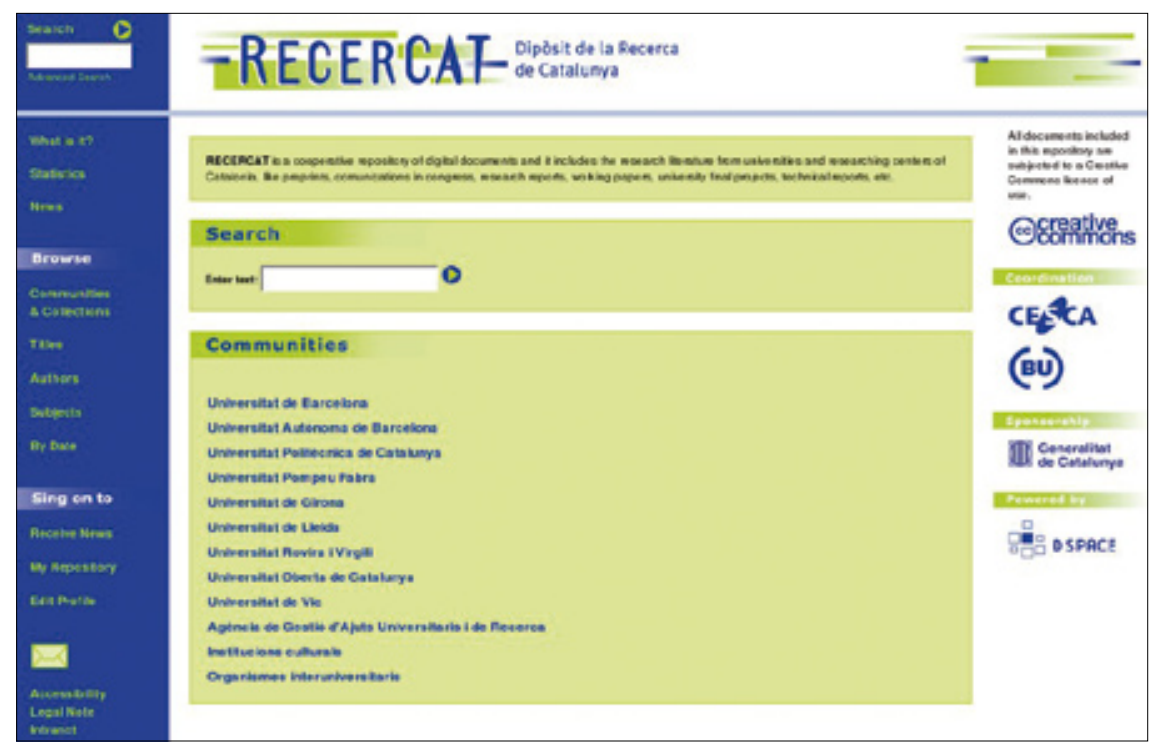

Dipòsit de la Recerca de Catalunya (Recercat)

positorio es importante la elección de software libre, que es el tipo de programas que más se está usando para estos sistemas. Si unimos los conceptos de acceso abierto y software libre obtenemos un binomio ganador (Vega, 2006), pero si además le añadimos el concepto de estándares abiertos (Corrado, 2005), necesario para una buena interoperatividad entre depósitos, obtenemos una apuesta robusta y segura para la creación de repositorios institucionales.

Existen varias aplicaciones libres para implementar estos productos. Aunque están en evolución continua -condición intrínseca del software libre-, pueden ser usadas en entornos de producción con resultados plenamente satisfactorios. $T D R$ está operativo desde 2001 y cuenta con 4.800 tesis de 17 universidades de diferentes comunidades autónomas españolas.

Recercat funciona desde 2005. En los primeros meses se llegó rápidamente a la cifra de 1.000 documentos de investigación y hoy ya cuenta con más de 3.800 de 15 instituciones. Un año más tarde llegaron $R A C O$ y Padicat. En el primero participan hasta 31 instituciones con 143 revistas y más de 38.000 artículos; el segundo ofrece 2.400 capturas de 810 sitios web, lo que significa 24 millones de ficheros (páginas web, imágenes, etc.).

Finalmente un apunte: este artículo se centra en tecnología, pero es importante no perder la perspectiva de que la creación de repositorios desde el punto de vista tecnológico no es un problema (CD-LOR). Como se ha descrito, existen muchas opciones más o menos desarrolladas que lo permiten. Y existen ejemplos en producción que lo demuestran, como son TDX, Recercat, $R A C O$ y Padicat. Sin embargo, hay otros aspectos que son más complejos, como dotarlos de contenidos, ponerse de acuerdo en los metadatos, establecer circuitos de trabajo, etc. Elaborar de forma cooperativa los repositorios ayuda en gran medida a alguno de estos aspectos, pues se pueden adoptar procedimientos comunes, desarrollar políticas de preservación a largo plazo y compartir los costes del desarrollo y su gestión. Además, el hecho de estar en un solo repositorio documentos de diferentes instituciones aumenta su visibilidad, consiguiendo de esta manera una mayor difusión de la investigación realizada y ayudando al desarrollo de la e-ciencia y de la sociedad de la información del país.

\section{Agradecimientos}

La creación de los repositorios que han servido de ejemplo a lo lar- 
go del artículo no hubiera sido posible sin el esfuerzo de mucha gente, tanto de dentro de los consorcios (Cesca y CBUC) como de fuera. De los consorcios, hay que citar a Marc Boix, Joan Cambras, Núria Comellas, Jesús Martín, Maite Novo, Jordi Prats, Sandra Reoyo, Ramon Ros, Marc Terrones, Xavier Torelló y Natalia Torres, además de los directores Miquel Huguet y Lluís Anglada, artífices de los proyectos. Fuera de los consorcios, la lista crece demasiado exponencialmente como para individualizar el agradecimiento, pues en cada institución participante en alguno de los repositorios hemos hallado la colaboración necesaria sin la cual no hubiera sido posible darles vida.

\section{Referencias}

Anglada, Lluís.; Reoyo-Tudó, Sandra; RosGomé, Ramon. "Los repositorios como componentes esenciales de las bibliotecas digitales: la experiencia de las bibliotecas universitarias de Cataluña". En: $3^{\circ}$ Jornada sobre la biblioteca digital universitaria, 2005.
Borràs, Antoni; Carreño, Juan; Jorba, Ferran Prats, Jordi; Ros, Ramon. "Programari de codi lliure per gestionar dipòsits digitals: el procés de tria dut a terme al CBUC". En: BiD: textos universitaris de biblioteconimia i documentació, 2006, junio, n. 16

CD-LOR. Community dimensions of learning objects repositories . Consultado en: 04-10-07. http://www.academy.gcal.ac.uk/cd-lor

Chawner, Brenda. "F/OSS in the library world an exploration". En: Open source application spaces: fifth workshop on open source software engineering (5-Wosse). ACM: St. Louis, 2005.

Corrado, Edward M. "The importance of open access, open source, and open standards for libraries". En: Issues in science and technology librarianship, 2005, n. 42. Consultado en: 04 10-07.

http://www.istl.org/05-spring/article2.html

Declaración de Berlín sobre el acceso abierto al conocimiento en el ámbito de las ciencias y las humanidades. Consultado en: 04-10-07.

http://www.zim.mpg.de/openaccess-berlin/berlin-declaration.html

Driver. The Driver guidelines. Consultado en 04-10-07.

http://www.driver-support.eu/en/guidelines.html

Gallart-Marsillas, Núria; Keefer, Alice. Preservació de dipòsits digitals, 20 marzo 2007. Consultado en: 04-10-07.

http://hdl.handle.net/2072/4078

Huguet, Miquel; Anglada, Lluís; De-la-VegaSivera, Ricard. "Catalan policies and experiences on cooperative repositories". En: 1st Iberian grid infrastructure conference (Ibergrid), 2007, pp. 63-75.

Llueca, Ciro. "Archivando la web, el proyecto Padicat (Patrimonio Digital de Cataluña)”. En: El profesional de la información, 2006, noviembrediciembre, v. 15 , n. 6 , pp. $473-478$

Mas-Hernández, Jordi. "Programari lliure: tècnicament viable, econòmicament sostenible socialment just”. Barcelona: Generalitat de Catalunya, Escola d'Administració Pública de Catalunya, 2006, 140, p. ISBN 84-393-6995-6.

Open Society Institute. A guide to institucional repository software. V. 3.0. Enero 2004. Consultado en: 04-10-07.

http://www.soros.org/openaccess/software

Sparc. The case for institutional repositories: a Sparc position paper. Consultado en: 04-10-07. http://www.arl.org/sparc/publications/papers.html

Tramullas, Jesús; Garrido-Picazo, Piedad. "Software libre para repositorios institucionales: propuestas para un modelo de evaluación de prestaciones". En: El profesional de la información, 2006, mayo-junio, v. 15, n. 3, pp. 171-181.

De-la-Vega-Sivera, Ricard. et al. "Dipòsits collectius d'e-informació". En: V Jornades de programari lliure, 2006. Consultado en: 04-10-07. http://hdl.handle.net/2072/2195

Ricard de-la-Vega-Sivera, Centre de Supercomputació de Catalunya, Barcelona.

rdelavega@cesca.es

\section{Próximos temas centrales}

\author{
Marzo 2008 \\ Mayo 2008 \\ Julio 2008 \\ Septiembre 2008 \\ Noviembre 2008
}

\author{
Innovación en bibliotecas públicas \\ Presente y futuro de la profesión \\ Libros electrónicos \\ Información en la empresa \\ Redes sociales
}

Los interesados pueden remitir notas, artículos, propuestas, publicidad, comentarios, etc., sobre estos temas a:

epi@elprofesionaldelainformacion.com 